\title{
Detecting the Diffuse Supernova Neutrino Background with LENA
}

\author{
R. Möllenberg, ${ }^{1,}$ | F. von Feilitzsch, ${ }^{2}$ D. Hellgartner, ${ }^{2}$ L. Oberauer, ${ }^{2}$ \\ M. Tippmann, ${ }^{2}$ J. Winter, ${ }^{3}$ M. Wurm ${ }^{3}$ and V. Zimmer ${ }^{2}$ \\ ${ }^{1}$ Excellence Cluster Universe, Technische Universität München, 85748 Garching, Germany \\ ${ }^{2}$ Physik Department, Technische Universität München, 85748 Garching, Germany \\ ${ }^{3}$ Institut für Physik, Excellence Cluster PRISMA, \\ Johannes Gutenberg Universität Mainz, 55128 Mainz, Germany
}

(Dated: September 13, 2018)

\begin{abstract}
LENA (Low Energy Neutrino Astronomy) has been proposed as a next generation 50 kt liquid scintillator detector. Its large target mass allows to search for the Diffuse Supernova Neutrino Background (DSNB), which was generated by the cumulative emissions of all core-collapse supernovae throughout the universe. Indistinguishable background from reactor and atmospheric electron antineutrinos limits the detection window to the energy range between $9.5 \mathrm{MeV}$ and $25 \mathrm{MeV}$. Depending on the mean supernova neutrino energy, about 5 to 10 events per year are expected in this energy window. The background from neutral current reactions of atmospheric neutrinos surpasses the DSNB by more than one order magnitude, but can be suppressed by pulse shape discrimination. Assuming that the residual background is known with $5 \%$ uncertainty, the DSNB can be detected with $3 \sigma$ significance after 10 years of data taking. In case that no hint for a signal is seen, current standard DSNB models would be ruled out with more than $90 \%$ C.L.
\end{abstract}

\section{INTRODUCTION}

The cumulative neutrino emissions of all core-collapse supernovae throughout the universe have generated the Diffuse Supernova Neutrino Background (DSNB). The DNSB contains information about the redshift dependent core-collapse supernova rate and about the average neutrino spectrum of a core-collapse supernova. Up to now the DSNB could not be detected due to the low flux. The best limit was achieved by the Super-Kamiokande experiment which sets an upper limit (90\% C.L.) on the flux of $3.1 \bar{\nu}_{\mathrm{e}} \mathrm{cm}^{-2} \mathrm{~s}^{-1}$ for $\mathrm{E}_{\bar{\nu}_{\mathrm{e}}}>17.3 \mathrm{MeV}$ 1], which is about a factor of two to four above the currently predicted values [1].

The DSNB contains neutrinos and antineutrinos of all flavours, but the detection channel with the largest cross section, the inverse beta decay channel $\left(\bar{\nu}_{\mathrm{e}}+\mathrm{p} \rightarrow \mathrm{e}^{+}+\mathrm{n}\right)$, is only sensitive to electron antineutrinos. Compared to Super-Kamiokande, the proposed LENA (Low Energy Neutrino Astronomy) detector [2] has the advantage that it can detect the $2.2 \mathrm{MeV}$ gamma of the neutron capture on hydrogen. Hence, the coincidence of the prompt positron and the delayed neutron signal can be used to suppress background events, which is crucial to detect the low DSNB flux.

The detection of the DSNB in LENA was analyzed in [3, neglecting the background from neutral current reactions of atmospheric neutrinos, which was observed by the KamLAND experiment [4] after the publication of 3 . In the present work, this important background is included for the first time and the calculations of the other backgrounds are updated. In Sec. II a brief description of the LENA detector is presented. The simulation

\footnotetext{
* randolph.moellenberg@ph.tum.de
}

of the DSNB and the corresponding background spectra is discussed in Sec. III and Sec. IV] As the background from neutral current reactions of atmospheric neutrinos surpasses the DSNB signal [4, an efficient reduction of this background is neccessary. Sec. $\mathrm{V}$ discusses how this can be achieved by pulse shape discrimination. Finally, the detection potential of the DSNB is discussed in Sec. VI.

\section{THE LENA DETECTOR}

Figure 1 shows a schematic overview of the currently proposed LENA design [5]. The neutrino target consists of $\sim 50 \mathrm{kt}$ of liquid scintillator based on linear-akylbenzene (LAB). The emitted light is detected by photomultiplier tubes (PMTs) that are mounted with nonimaging light concentrators (LCs) inside individual pressure encapsulations that are filled with a non-scintillating buffer liquid. The apertures of these optical modules face the boundary of the target volume at a radius of $14 \mathrm{~m}$. The corresponding effective optical coverage is $\sim 30 \%$. The radius of the cylindrical concrete tank is $16 \mathrm{~m}$, so that the target volume is shielded by $2 \mathrm{~m}$ of liquid scintillator. A muon veto formed by gas detectors is placed above the detector tank and provides auxiliary information for the reconstruction of cosmic muon tracks. In order to identify and reconstruct also inclined muon tracks, an instrumented water volume surrounding the tank serves as an active Water-Črenkov muon veto. Additionally, it shields the target volume from fast neutrons.

The preferred location for the detector is the Pyhäsalmi mine in Finland, that has $1400 \mathrm{~m}$ of rock coverage, corresponding to a shielding of $4000 \mathrm{~m}$ water equivalent (w.e.). Hence, the cosmic muon flux is reduced to $\sim 0.2 \mathrm{~m}^{-2} \mathrm{~h}^{-1}[6$. 


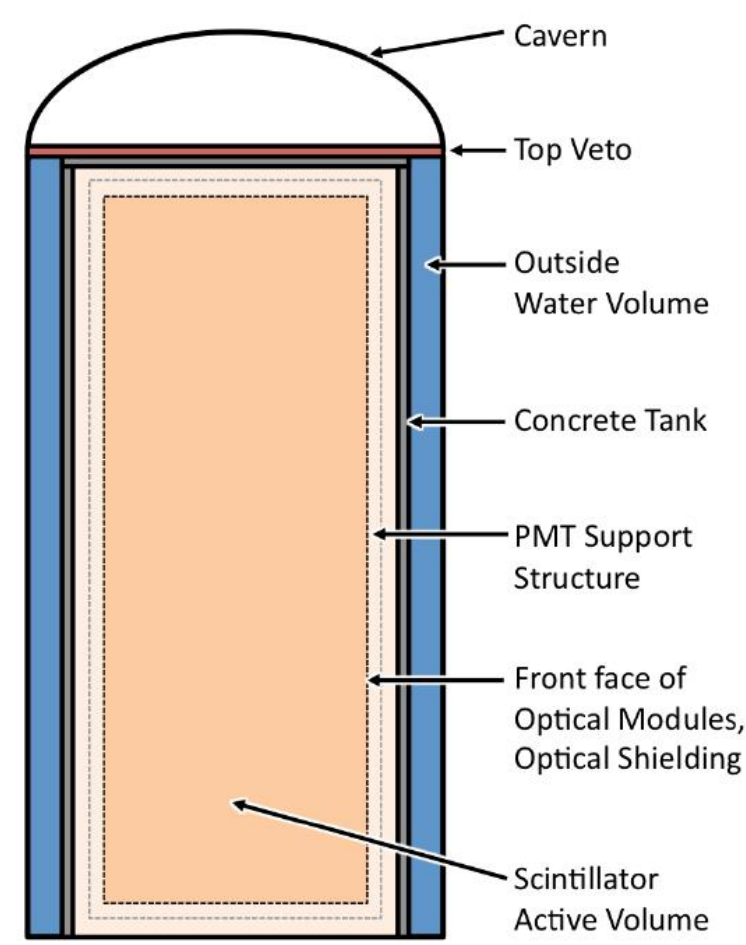

FIG. 1. Schematical view of the LENA detector [5].

\section{SIMULATION OF THE DIFFUSE SUPERNOVA NEUTRINO BACKGROUND SPECTRUM}

The detection channel for the DSNB is the inverse beta decay (IBD) reaction of an $\bar{\nu}_{e}$ on a free proton $\left(\bar{\nu}_{\mathrm{e}}+\mathrm{p} \rightarrow \mathrm{e}^{+}+\mathrm{n}\right)$, as it has the largest cross section at low energies. Due to the kinematics of the IBD reaction, the kinetic energy of the neutron can be neglected [7. Thus, the kinetic energy of the positron is almost equal to the incident neutrino energy, reduced by the 1.8 MeV Q-Value of the IBD reaction. Hence, the neutrino energy can be reconstructed from the detected visible energy $\left(\mathrm{E}_{\nu} \approx \mathrm{E}_{\mathrm{vis}}+0.8 \mathrm{MeV}\right)$, considering that the positron annihilates with an electron into two $511 \mathrm{keV}$ gammas. The produced neutron gets captured by a proton with a mean capture time of $\sim 250 \mu \mathrm{s}$, producing a $2.2 \mathrm{MeV}$ gamma. Hence, the delayed coincidence between the prompt positron and the delayed neutron signal can be used to reject background events.

Assuming that the supernova neutrino spectrum and the luminosity is independent of the neutrino flavour such that $\left\langle\mathrm{E}_{\bar{\nu}_{\mathrm{e}}}\right\rangle=\left\langle\mathrm{E}_{\bar{\nu}_{\mu}}\right\rangle=\left\langle\mathrm{E}_{\bar{\nu}_{\tau}}\right\rangle$ and $\mathrm{L}_{\bar{\nu}_{\mathrm{e}}}=\mathrm{L}_{\bar{\nu}_{\mu}}=\mathrm{L}_{\bar{\nu}_{\tau}}=0.5 \cdot 10^{53} \mathrm{erg}$, neutrino oscillations can be neglected.

Thus, the detected DSNB spectrum is given by:

$$
\frac{\mathrm{dR}_{\nu}}{\mathrm{dE}_{\nu}}=\frac{\mathrm{dF}_{\nu}}{\mathrm{dE}_{\nu}} \cdot \sigma_{\nu}\left(\mathrm{E}_{\nu}\right) \cdot \mathrm{N}_{\mathrm{p}}
$$

where $\frac{\mathrm{dF}_{\nu}}{\mathrm{dE}_{\nu}}$ is the DSNB flux, $\sigma_{\nu}\left(\mathrm{E}_{\nu}\right)$ is the energy dependent cross section of the IBD reaction [7] and $\mathrm{N}_{\mathrm{p}}=3.67 \cdot 10^{33}$ is the number of protons in the target volume $(\mathrm{r}<13.5 \mathrm{~m},|\mathrm{z}|<48.5 \mathrm{~m}) \sqrt{1}[9$.

The DSNB flux is calculated with the following expression [10]:

$$
\frac{\mathrm{dF}_{\nu}}{\mathrm{dE}_{\nu}}=\frac{\mathrm{c}}{\mathrm{H}_{0}} \int_{0}^{\mathrm{z}_{\mathrm{m}}} \frac{\mathrm{dN}_{\nu}\left(\mathrm{E}_{\nu}^{\prime}\right)}{\mathrm{dE}_{\nu}^{\prime}} \frac{\mathrm{R}_{\mathrm{SN}}(\mathrm{z}) \mathrm{dz}}{\sqrt{\Omega_{\mathrm{m}}(1+\mathrm{z})^{3}+\Omega_{\Lambda}}}
$$

where $R_{\mathrm{SN}}(\mathrm{z})$ is the supernova rate at redshift $\mathrm{z}$, $\mathrm{E}_{\nu}^{\prime}=\mathrm{E}_{\nu}(1+\mathrm{z})$ is the redshift corrected energy of the neutrinos, $\frac{\mathrm{dN}_{\nu}\left(\mathrm{E}_{\nu}\right)}{\mathrm{dE}_{\nu}}$ is the mean number spectrum of the neutrinos emitted by one supernova explosion, $\mathrm{H}_{0}$ is the Hubble constant, $\mathrm{c}$ is the speed of light, $\Omega_{\mathrm{m}}$ is the cosmic matter density, $\Omega_{\Lambda}$ is the cosmic constant and $\mathrm{z}_{\mathrm{m}}=5$ is the redshift where the first core-collapse supernova occurred.

Assuming that every star above 8 solar masses ends in a core-collapse supernova and using the Salpeter initial mass function [11, $\mathrm{R}_{\mathrm{SN}}(\mathrm{z})$ can be derived from the star formation rate $\mathrm{R}_{*}(\mathrm{z})$ to be $\mathrm{R}_{\mathrm{SN}}(\mathrm{z})=0.0122 \mathrm{M}_{\odot}^{-1} \mathrm{R}_{*}(\mathrm{z})$.

The used parametrization for the star formation rate is 10$]$ :

$$
\begin{aligned}
\mathrm{R}_{*}(\mathrm{z})= & 0.32 \mathrm{f}_{\mathrm{SN}} \frac{\mathrm{H}_{0}}{70 \mathrm{kms}^{-1} \mathrm{Mpc}^{-1}} \frac{\mathrm{e}^{3.4 \mathrm{z}}}{\mathrm{e}^{3.8 \mathrm{z}}+45} \\
& \frac{\sqrt{\Omega_{\mathrm{m}}(1+\mathrm{z})^{3}+\Omega_{\Lambda}}}{(1+\mathrm{z})^{3 / 2}} \mathrm{yr}^{-1} \mathrm{Mpc}^{-3}
\end{aligned}
$$

where $\mathrm{f}_{\mathrm{SN}}=1.5 \pm 0.3$ is a normalization factor, such that $\mathrm{R}_{\mathrm{SN}}(\mathrm{z}=0)=1.25 \cdot 10^{-4} \mathrm{y}^{-1} \mathrm{Mpc}^{-3}[12$.

The neutrino emission spectrum $\frac{\mathrm{dN}_{\nu}\left(\mathrm{E}_{\nu}\right)}{\mathrm{dE}_{\nu}}$ is approximated by a Maxwell-Boltzmann spectrum [13. The assumed mean supernova neutrino energy $\left\langle\mathrm{E}_{\nu}\right\rangle$ is in the range between $12 \mathrm{MeV}$ and $21 \mathrm{MeV}$, according to indirect constraints from chemical abundances of neutrino induced elements [14, which are in agreement with numerical supernova simulations [15 17.

Using equation 1, four different DSNB spectra with $\left\langle\mathrm{E}_{\nu}\right\rangle$ ranging from $12 \mathrm{MeV}$ to $21 \mathrm{MeV}$ were obtained from a GEANT4 18 based Monte Carlo simulation of the LENA detector [8]. The events were homogeneously distributed over the target volume, so that possible position dependent effects are considered.

Figure 2 shows the resulting DSNB spectra. The spectra peak at energies between $\sim 8 \mathrm{MeV}$ and $\sim 16 \mathrm{MeV}$, depending on $\left\langle\mathrm{E}_{\nu}\right\rangle$ and decrease exponentially at higher energies. The energy of the peak is lower than the corresponding $\left\langle\mathrm{E}_{\nu}\right\rangle$, because the neutrino energy is redshifted. After 10 years of data taking, about 80 to 150 DSNB events are expected. Thus, very low background levels are needed for a detection of the DSNB.

\footnotetext{
${ }^{1}$ Note that the volume directly infront of the optical modules can not be used due to a very inhomogeneous detector response in this area $[8$.
} 


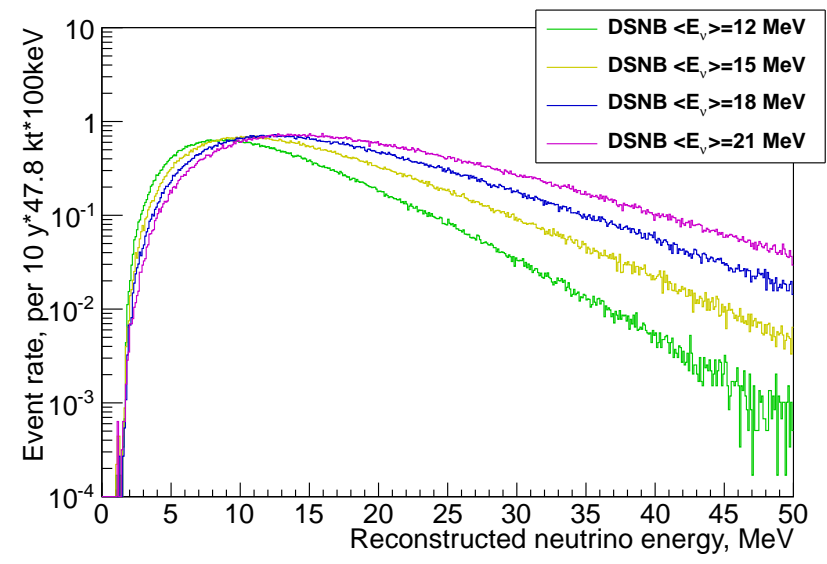

FIG. 2. The expected DSNB spectra in LENA for different supernova neutrino spectra with mean energies ranging from $12 \mathrm{MeV}$ to $21 \mathrm{MeV}$.

\section{BACKGROUNDS}

The background for the DSNB detection can be divided into three categories: Indistinguishable background from other $\bar{\nu}_{\mathrm{e}}$ sources, muon induced backgrounds and the neutral current reactions of atmospheric neutrinos.

\section{A. $\quad \bar{\nu}_{\mathrm{e}}$ Charged Current Background}

Other $\bar{\nu}_{\mathrm{e}}$ sources in the relevant energy region pose a dangereous background for the DSNB detection, as these events are indistinguishable from DSNB events. Possible $\bar{\nu}_{\mathrm{e}}$ sources are reactor neutrinos and atmospheric neutrinos.

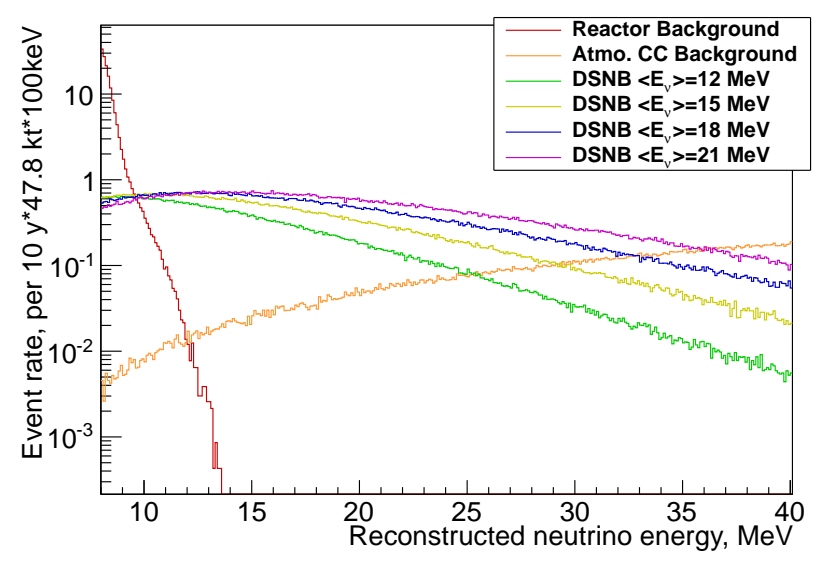

FIG. 3. The reactor and atmospheric $\bar{\nu}_{\mathrm{e}}$ spectrum expected for LENA at Pyhäsalmi. For comparison, the DSNB spectra with $\left\langle\mathrm{E}_{\nu}\right\rangle$ ranging from $12 \mathrm{MeV}$ to $21 \mathrm{MeV}$ are depicted.

The reactor and atmospheric $\bar{\nu}_{\mathrm{e}}$ flux at the Pyhäsalmi location were calculated in [19. Based on these results, the reactor and atmospheric $\bar{\nu}_{\mathrm{e}}$ spectra were simulated analogous to the DSNB signal. Figure 3 shows the resulting reactor and atmospheric background spectra. Below about $9.5 \mathrm{MeV}$, the reactor $\bar{\nu}_{\mathrm{e}}$ spectrum surpasses the DSNB spectra and decreases exponentially with rising energy. The atmospheric $\bar{\nu}_{\mathrm{e}}$ background is suppressed at low energies and rises with the energy until it surpasses the DSNB spectrum for $\left\langle\mathrm{E}_{\nu}\right\rangle=12 \mathrm{MeV}$ at $\sim 25 \mathrm{MeV}$ reconstructed neutrino energy. Hence, the detection window is set to $9.5 \mathrm{MeV}-25 \mathrm{MeV}$, which further reduces the event rate to between 50 and 100 events in $10 \mathrm{y}$. About 11 remaining reactor and atmospheric $\bar{\nu}_{e}$ events are expected in the detection window.

\section{B. Muon-induced Backgrounds}

When a cosmic muon traverses the target volume, it can produce radioactive isotopes by spallation reactions on carbon. The majority of these radioisotopes can be vetoed by the delayed coincidence condition. However, ${ }^{9} \mathrm{Li}\left(\mathrm{Q}_{\beta^{-}}=13.6 \mathrm{MeV}\right)$ can $\beta^{-}$decay into excited states of ${ }^{9} \mathrm{Be}$, leading to the emission of a neutron 20. About $10^{3}{ }^{9} \mathrm{Li} \beta-\mathrm{n}$ events are expected per $10 \mathrm{y}$ in the detection window. ${ }^{9} \mathrm{Li}$ has a short life time $(257.2 \mathrm{~ms})$ and is produced close to the muon track. Thus, the ${ }^{9} \mathrm{Li}$ background can be reduced to less than 0.01 events per $10 \mathrm{y}$, by vetoing a cylinder with $2 \mathrm{~m}$ radius around each muon track for $2.5 \mathrm{~s}$ which introduces $0.2 \%$ effective dead time [8.

Another possible background are fast neutrons which are produced in the surrounding rock by cosmic muons. A small fraction of these neutrons propagate into the target volume, without triggering the Water-Čerenkov muon veto. Inside the target volume, the neutrons cause a prompt signal by scattering reactions on hydrogen and carbon. Afterwards, they thermalize and are finally captured by a proton, thus mimicking the delayed coincidence signature. The fast neutron background was simulated with the GEANT4-based Monte Carlo simulation, assuming a mean cosmic muon energy of $300 \mathrm{GeV}$ [6]. It was found that the fast neutron rate in the target volume is about one order of magnitude larger than the DSNB event rate. But as the fast neutrons enter the detector from outside, they are not homogeneously distributed over the target volume. Instead, the majority of the fast neutrons are events close to the boundary of the target volume. Thus, it is possible to lower the fast neutron background to $4.9 \pm 0.4$ (stat.) events per $10 \mathrm{y}$, by reducing the fiducial volume radius to $11.0 \mathrm{~m}$. This cut reduces the DSNB event rate by $34 \%$, so that only 32.4 events per $10 \mathrm{y}$ are expected for $\left\langle\mathrm{E}_{\nu}\right\rangle=12 \mathrm{MeV}$. Another possible method to reduce this background is by pulse shape analysis (see Sec. V). 


\section{Neutral Current Atmospheric $\nu$ Background}

Additional to the CC background of atmospheric $\bar{\nu}_{\mathrm{e}}$, $\mathrm{NC}$ reactions of atmospheric neutrinos and antineutrinos of all flavours pose a background for the DSNB detection. This NC background was first observed by the KamLAND experiment 4] and was not included in previous analyses [3, 19. Several neutrino reactions contribute to this background. They all have in common that a single neutron is emitted (e.g. $\nu_{\mathrm{x}}+{ }^{12} \mathrm{C} \rightarrow \nu_{\mathrm{x}}+\mathrm{n}+{ }^{11} \mathrm{C}$, though other more complex reactions are also possible), which mimics the IBD event signature (see Section IV B).

In order to simulate this background, the reactions of atmospheric neutrinos inside the target volume were simulated with the GENIE Neutrino Monte-Carlo Generator (Version 2.6.6) 21, using the Bartol atmospheric neutrino fluxes 22 as input. As these fluxes were calculated for the Super-Kamiokande experiment, the resulting event rates have to be scaled by a factor of two in order to consider the higher geographic latitude of the Pyhäsalmi location 19. After simulating the neutrino interaction, the final state particles were tracked with the GEANT4-based simulation of the LENA detector. Figure 4 shows the resulting atmospheric NC background spectrum.

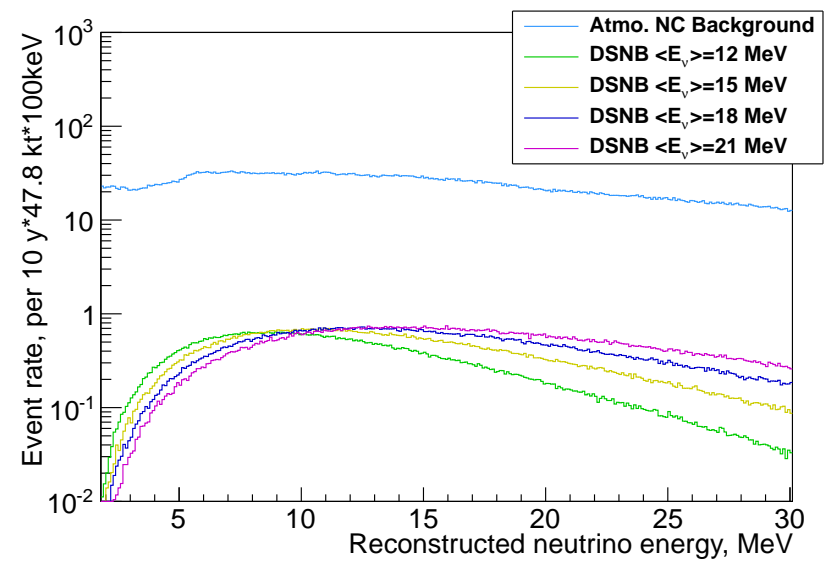

FIG. 4. The simulated atmospheric neutrino NC background spectrum. For comparison, the DSNB spectra with $\left\langle\mathrm{E}_{\nu}\right\rangle$ ranging from $12 \mathrm{MeV}$ to $21 \mathrm{MeV}$ are also depicted.

The NC background spectrum surpasses the expected DSNB spectra by more than one order of magnitude over the whole energy region. Overall, $3.27 \cdot 10^{3}$ events per $10 \mathrm{y}$ are expected in the DSNB detection window ${ }^{2}$ Hence, an efficient method to supress this background is necessary in order to detect the DSNB.

A possible background suppression method is to look for the coincidence of an atmospheric neutrino NC event

\footnotetext{
${ }^{2}$ Considering the larger atmospheric neutrino flux, this result is in agreement with the KamLAND measurement [4.
}

\begin{tabular}{|l|c|}
\hline Reaction channel & Branching ratio \\
\hline$(1) \nu_{\mathrm{x}}+{ }^{12} \mathrm{C} \rightarrow \nu_{\mathrm{x}}+\mathrm{n}+{ }^{11} \mathrm{C}$ & $38.8 \%$ \\
$(2) \nu_{\mathrm{x}}+{ }^{12} \mathrm{C} \rightarrow \nu_{\mathrm{x}}+\mathrm{p}+\mathrm{n}+{ }^{10} \mathrm{~B}$ & $20.4 \%$ \\
$(3) \nu_{\mathrm{x}}+{ }^{12} \mathrm{C} \rightarrow \nu_{\mathrm{x}}+2 \mathrm{p}+\mathrm{n}+{ }^{9} \mathrm{Be}$ & $15.9 \%$ \\
$(4) \nu_{\mathrm{x}}+{ }^{12} \mathrm{C} \rightarrow \nu_{\mathrm{x}}+\mathrm{p}+\mathrm{d}+\mathrm{n}+{ }^{8} \mathrm{Be}$ & $7.1 \%$ \\
$(5) \nu_{\mathrm{x}}+{ }^{12} \mathrm{C} \rightarrow \nu_{\mathrm{x}}+\alpha+\mathrm{p}+\mathrm{n}+{ }^{6} \mathrm{Li}$ & $6.6 \%$ \\
$(6) \nu_{\mathrm{x}}+{ }^{12} \mathrm{C} \rightarrow \nu_{\mathrm{x}}+2 \mathrm{p}+\mathrm{d}+\mathrm{n}+{ }^{7} \mathrm{Li}$ & $1.3 \%$ \\
$(7) \nu_{\mathrm{x}}+{ }^{12} \mathrm{C} \rightarrow \nu_{\mathrm{x}}+3 \mathrm{p}+2 \mathrm{n}+{ }^{7} \mathrm{Li}$ & $1.2 \%$ \\
$(8) \nu_{\mathrm{x}}+{ }^{12} \mathrm{C} \rightarrow \nu_{\mathrm{x}}+\mathrm{d}+\mathrm{n}+{ }^{9} \mathrm{~B}$ & $1.2 \%$ \\
$(9) \nu_{\mathrm{x}}+{ }^{12} \mathrm{C} \rightarrow \nu_{\mathrm{x}}+2 \mathrm{p}+\mathrm{t}+\mathrm{n}+{ }^{6} \mathrm{Li}$ & $1.1 \%$ \\
$(10) \nu_{\mathrm{x}}+{ }^{12} \mathrm{C} \rightarrow \nu_{\mathrm{x}}+\alpha+\mathrm{n}+{ }^{7} \mathrm{Be}$ & $1.1 \%$ \\
$(11) \nu_{\mathrm{x}}+{ }^{12} \mathrm{C} \rightarrow \nu_{\mathrm{x}}+3 \mathrm{p}+\mathrm{n}+{ }^{8} \mathrm{Li}$ & $1.1 \%$ \\
\hline other reaction channels & $4.2 \%$ \\
\hline
\end{tabular}

TABLE I. The branching ratios of the different atmospheric NC background channels in the DSNB detection window.

with the subsequent decay of any produced radioactive isotopes. Table $\mathrm{I}$ shows the branching ratios of the different atmospheric NC background channels in the DSNB detection window. ${ }^{11} \mathrm{C}$ is produced with a branching ratio of about $39 \%$. In this case it is possible to veto the atmospheric $\mathrm{NC}$ event by looking for the coincidence of the IBD-like event and the subsequent decay of ${ }^{11} \mathrm{C}$. As the lifetime of ${ }^{11} \mathrm{C}$ is $\tau=29.4 \mathrm{~min}$, this cut can only be applied if the fiducial volume is reduced to $30 \mathrm{kt}$, in order to prevent accidental coincidences of IBD events and background events from external gamma rays. But except ${ }^{8} \mathrm{Li}$ which is produced in reaction $(11)$, the other produced isotopes are either stable $\left({ }^{10} \mathrm{~B},{ }^{9} \mathrm{Be},{ }^{7} \mathrm{Li},{ }^{6} \mathrm{Li}\right)$, have a too long life time $\left({ }^{7} \mathrm{Be}, \tau=76.9 \mathrm{~d}\right)$ or decay almost instantly $\left({ }^{9} \mathrm{~B},{ }^{8} \mathrm{Be}\right)$.

Hence, the atmospheric NC background can only be reduced by about $40 \%$ by looking for the coincident decay of a radioactive isotope. Thus, a more efficient background suppression method is needed. A possible option is to reject atmospheric neutrino $\mathrm{NC}$ events by analyzing their pulse shape. This method will be discussed in the next Section.

\section{PULSE SHAPE DISCRIMINATION OF BACKGROUND EVENTS}

Heavier particles, like protons, neutrons or alphas, have a different pulse shape than positrons. Figure 5 shows a comparison between the average neutron and gamma pulse shape as measured in a small scale laboratory experiment at the Maier-Leibnitz-Laboratorium (MLL) in Garching, Germany 23. A clear difference between the two pulse shapes is visible. Hence, this difference can be used to distinguish neutron from positron events. The neutron pulse shape was parametrized by the following PDF:

$$
\mathrm{F}(\mathrm{t})=\sum_{\mathrm{i}} \frac{\mathrm{N}_{\mathrm{i}}}{\tau_{\mathrm{i}}} \mathrm{e}^{-\frac{\mathrm{t}}{\tau_{\mathrm{i}}}}
$$




\begin{tabular}{|c|c|c|c|}
\hline Parameter & Electrons/Positrons & Protons & Alphas \\
\hline $\mathrm{N}_{1}$ & 0.67 & 0.61 & 0.44 \\
$\mathrm{~N}_{2}$ & 0.19 & 0.21 & 0.16 \\
$\mathrm{~N}_{3}$ & 0.14 & 0.18 & 0.40 \\
$\tau_{1}$ & $6.8 \mathrm{~ns}$ & $7.0 \mathrm{~ns}$ & $3.2 \mathrm{~ns}$ \\
$\tau_{2}$ & $26.5 \mathrm{~ns}$ & $27.3 \mathrm{~ns}$ & $18 \mathrm{~ns}$ \\
$\tau_{3}$ & $152.3 \mathrm{~ns}$ & $140.3 \mathrm{~ns}$ & $190 \mathrm{~ns}$ \\
$\mathrm{k}_{\mathrm{b}}$ & $0.15 \frac{\mathrm{mm}}{\mathrm{MeV}}$ & $0.12 \frac{\mathrm{mm}}{\mathrm{MeV}}$ & $0.11 \frac{\mathrm{mm}}{\mathrm{MeV}}$ \\
\hline
\end{tabular}

TABLE II. The photon emission parameters for electrons, protons and alphas [23, 25]. The $\mathrm{k}_{\mathrm{b}}$ value for protons was taken from a calibration measurement in the Borexino experiment, which uses PC as scintillator [26].

\begin{tabular}{|c|c|}
\hline Parameter & Value \\
\hline Rayleigh scattering length & $40 \mathrm{~m}$ \\
Absorption-reemission length & $60 \mathrm{~m}$ \\
Absorption length & $20 \mathrm{~m}$ \\
\hline
\end{tabular}

TABLE III. The values for the scattering and absorption lengths [19] which were used in the simulation. As the absorption length can not be measured directly, it was set to a value that is in agreement with the attenuation length measurements that were performed in [27].

which was used as an input value for Monte-Carlo simulation of the LENA detector. Table II shows the parameters of the photon emission PDF that were used for the simulation of electrons, positrons, protons and alphas. The used scattering and absorption lengths are denoted in table III.

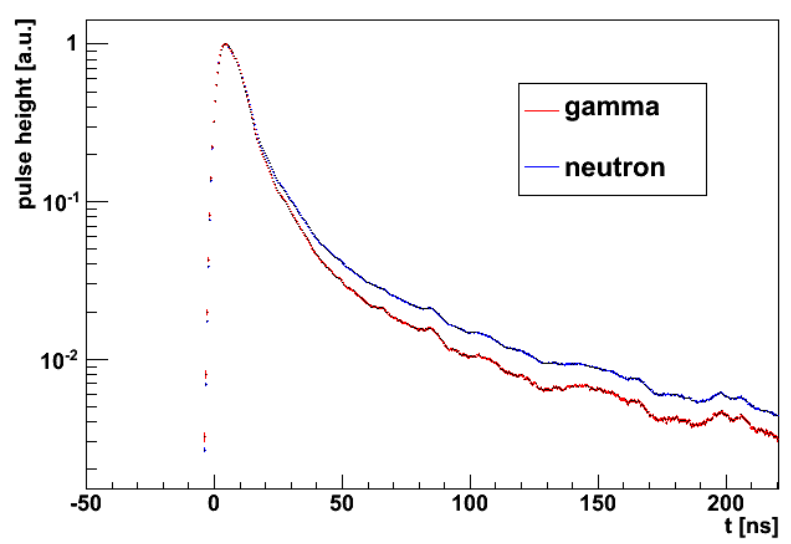

FIG. 5. Average normalized pulses for gamma (denoted in red) and neutron (denoted in blue) events [23].

Figure 6 shows a comparison between the average pulse shapes of neutron and IBD events in the center of the detector. The average visible energy of the events was $9.2 \mathrm{MeV}$, which corresponds to a $\bar{\nu}_{\mathrm{e}}$ with $10 \mathrm{MeV}$. Compared to the small scale laboratory experiment, the difference between the two pulse shapes is much smaller. The reason for this effect is that a large fraction of the emitted photons are scattered before they are detected by a PMT, which distorts the pulse shape. Nevertheless, a clear difference between the two pulse shapes is still visible, that can be used to identify fast neutron and atmospheric NC events.

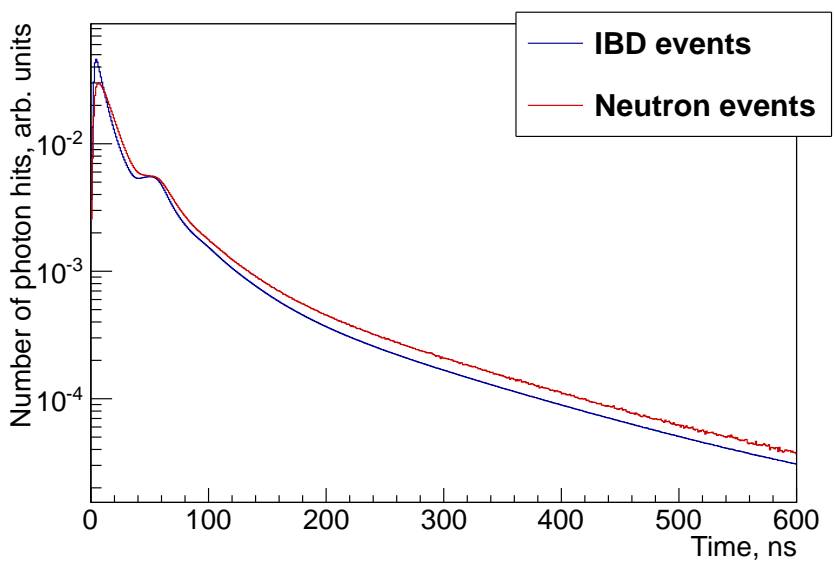

FIG. 6. Comparison between the average normalized pulse shape of IBD (denoted in blue) and neutron (denoted in red) events in the center of LENA with $\mathrm{E}_{\mathrm{vis}}=9.2 \mathrm{MeV}$.

For the pulse shape analysis, two different methods were used. In the tail-to-total method, the photon signal is integrated over two different intervals. One interval includes the complete pulse, the so called total interval, and the other one encompasses the last part of the signal, the tail interval. Subsequently, the ratio between the tail and the total interval is calculated.

Another more complex method to discriminate between two different particle types is the gatti method 28. First of all, the average pulse shapes for the two particles are calculated from events where the particle type is known. Subsequently, a set of weights $\mathrm{P}_{\mathrm{i}}$ is calculated from the two pulse shapes:

$$
\mathrm{P}_{\mathrm{i}}=\frac{\alpha_{\mathrm{i}}-\beta_{\mathrm{i}}}{\alpha_{\mathrm{i}}+\beta_{\mathrm{i}}},
$$

where $\alpha_{i} / \beta_{i}$ are the normalized number of photons detected in bin i of the $\alpha / \beta$ signal. In the last step the so called gatti parameter $G_{S}$ for a normalized signal $S_{i}$ from an unknown particle is calculated:

$$
\mathrm{G}_{\mathrm{S}}=\sum_{\mathrm{i}} \mathrm{P}_{\mathrm{i}} \mathrm{S}_{\mathrm{i}}
$$

Due to the weigths $P_{i}$, which are determined by the average pulse shapes of the two particles, the value of the gatti parameter depends on the particle type.

Figure 7 shows the tail-to-total ratio and gatti parameter distribution for IBD and neutron events. For the tailto-total ratio distribution, a clear difference between IBD and neutron events is visible, while there is also an overlap. Hence, an efficient discrimination is only possible for 

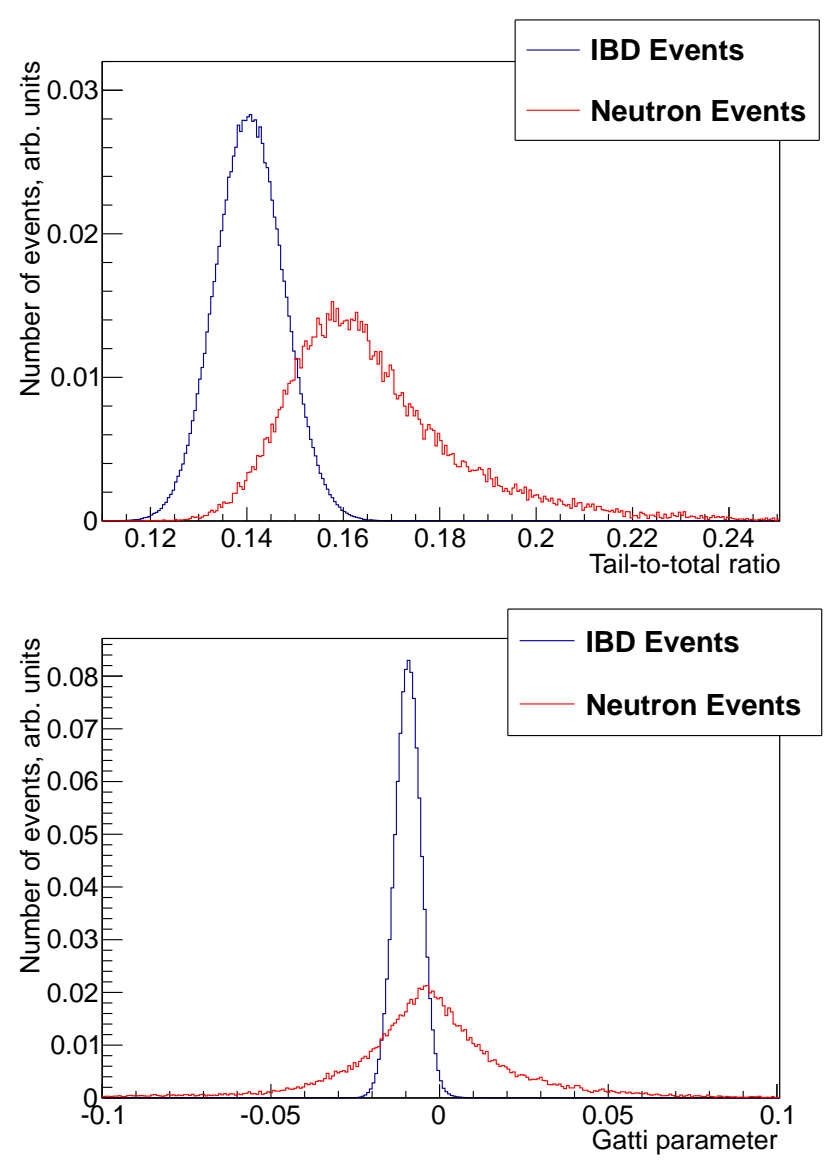

FIG. 7. The tail-to-total ratio (upper plot) and gatti parameter (lower plot) distribution for IBD (denoted in blue) and neutron events (denoted in red) in the center of LENA with $\mathrm{E}_{\mathrm{vis}}=9.2 \mathrm{MeV}$.

a low acceptance of IBD events. The gatti parameter distribution shows a large overlap between IBD and neutron events. The reason for this is that there are large fluctuations of the indivual neutron pulse shapes, as neutrons can make elastic scattering reactions on protons as well as inelastic scattering reactions on carbon, which lead to a different pulse shape. Hence, the gatti weights are not optimized for each of the possible reactions. Nevertheless, it was found that the gatti method performs well on some pulses, where the tail-to-total method does not perform well. Hence, the overall discrimination efficiency can be enhanced by applying both the tail-to-total and the gatti method.

Table IV shows the atmospheric NC and fast neutron background rate, depending on the acceptance for IBD events of the used pulse shape cut for $44 \mathrm{kt}$ fiducial volume. In case that a strict pulse shape cut is used, which only accepts $40 \%$ of all IBD events, the atmospheric NC background is reduced by two orders of magnitude to $21.8 \pm 0.4$ (stat.) events per $10 \mathrm{y}$. This cut also reduces the fast neutron rate to $1.8 \pm 0.2$ (stat.) events per $10 \mathrm{y}$, so that no further fiducial volume cut needs to be applied.

\begin{tabular}{|c|c|c|c|}
\hline $\begin{array}{c}\text { IBD } \\
\text { acceptance }\end{array}$ & $\begin{array}{c}\text { Atmospheric } \\
\text { NC rate }[10 \mathrm{y}]\end{array}$ & $\begin{array}{c}\text { Fast neutron } \\
\text { rate }[10 \mathrm{y}]\end{array}$ & $\begin{array}{c}\text { DSNB Signal } \\
\left\langle\mathrm{E}_{\nu}\right\rangle=12 \mathrm{MeV}\end{array}$ \\
\hline $90.0 \%$ & $378 \pm 2$ (stat.) & $8.6 \pm 0.5$ (stat.) & 40.2 \\
$80.0 \%$ & $155 \pm 1$ (stat.) & $4.5 \pm 0.4$ (stat.) & 35.8 \\
$50.0 \%$ & $34.4 \pm 0.5$ (stat.) & $2.1 \pm 0.3$ (stat.) & 22.4 \\
$40.0 \%$ & $21.8 \pm 0.4$ (stat.) & $1.8 \pm 0.2$ (stat.) & 17.9 \\
\hline
\end{tabular}

TABLE IV. The atmospheric NC and fast neutron background depending on the IBD acceptance of the used pulse shape cut for $44 \mathrm{kt}$ fiducial volume. For comparison, the corresponding DSNB event rates for $\left\langle\mathrm{E}_{\nu}\right\rangle=12 \mathrm{MeV}$ are also denoted.

\begin{tabular}{|c|c|}
\hline Background source & Rate $[10 \mathrm{y}]$ \\
\hline Reactor neutrinos & 2.0 \\
Atmospheric $\bar{\nu}_{e}$ & 2.2 \\
${ }^{9} \mathrm{Li} \beta^{-}-n$ & $<0.01$ \\
Fast neutrons & 1.8 \\
Atmospheric NC & 21.8 \\
\hline Sum & 27.8 \\
DSNB $\left(\left\langle\mathrm{E}_{\nu}\right\rangle=12 \mathrm{MeV}\right)$ & 17.9 \\
\hline
\end{tabular}

TABLE V. The expected background rates in the DSNB detection window after applying the pulse shape and the fiducial volume cut.

\section{DETECTION POTENTIAL}

Table $\mathrm{V}$ summarizes the contribution of the different background sources after applying the pulse shape and the fiducial volume cut. Overall, 27.8 background events per $10 \mathrm{y}$ are expected inside the detection window, while the predicted DSNB rate ranges from 17.9 $\left(\left\langle\mathrm{E}_{\nu}\right\rangle=12 \mathrm{MeV}\right)$ to $35.2\left(\left\langle\mathrm{E}_{\nu}\right\rangle=21 \mathrm{MeV}\right)$ events per $10 \mathrm{y}$.

A prerequisite for a positive detection of the DSNB is that the expected background rate is determined with a high precision. The reactor and atmospheric $\bar{\nu}_{\mathrm{e}}$ rate can be extrapolated from the measured rate outside the detection window. As the fast neutron rate decreases with the radius of the fiducial volume, it can be determined by analyzing the dependence of the event rate on the radius of the reconstructed position.

Measureing the atmospheric NC event rate is challenging, as the efficiency of the applied pulse shape cut must be known with high precision. A possible option is to look for events in the center of the detector, where two neutron captures were detected, as these events can only be due to atmospheric neutrino $\mathrm{NC}$ reactions. While the efficiency for IBD-like atmospheric NC events cannot be calculated directly from these events, they can be used to validate the Monte-Carlo simulation. Subsequently, the atmospheric NC event rate can be calculated with this validated Monte-Carlo simulation.

It is not clear how precise the background measurement will be. Hence, two scenarios are dicussed in following. An optimistic scenario of $5 \%$ background un- 


\begin{tabular}{|c|c|c|}
\hline$\left\langle\mathrm{E}_{\nu}\right\rangle$ & $\begin{array}{c}5 \% \text { background } \\
\text { uncertainty }\end{array}$ & $\begin{array}{c}25 \% \text { background } \\
\text { uncertainty }\end{array}$ \\
\hline $12 \mathrm{MeV}$ & $3.0 \sigma$ & $1.9 \sigma$ \\
$15 \mathrm{MeV}$ & $4.0 \sigma$ & $2.6 \sigma$ \\
$18 \mathrm{MeV}$ & $4.9 \sigma$ & $3.1 \sigma$ \\
$21 \mathrm{MeV}$ & $5.4 \sigma$ & $3.5 \sigma$ \\
\hline
\end{tabular}

TABLE VI. The expected detection significance after 10 years for different DSNB models with $\left\langle\mathrm{E}_{\nu}\right\rangle$ ranging from $12 \mathrm{MeV}$ to $21 \mathrm{MeV}$, assuming that the expected background rates are known with 5 and $25 \%$ precision, respectively.

certainty and pessimistic one of $25 \%$ background uncertainty. In order to assess the expected significance for a positive detection of the DSNB, it was assumed that the number of detected events equals the sum of the expected signal and background rate. From the number of detected events, background rate and background uncertainty, the confidence interval for the DSNB rate is calculated according to [29]. By increasing the size of the confidence interval such that the lower limit is almost zero, the significance of the DSNB detection is calculated. Table VI shows the resulting detection significance. In the optimistic case that the background uncertainty is $5 \%$, the DSNB can be detected with $3 \sigma$ significance in all cases. If the background is known with $25 \%$ precision, a $3 \sigma$ detection is only possible for $\left.\left\langle\mathrm{E}_{\nu}\right\rangle\right\rangle=18 \mathrm{MeV}$. Hence, the background needs to be well understood in order to detect the DSNB.

Figure 8 shows the DSNB detection potential, depending on the supernova rate $\mathrm{R}_{\mathrm{SN}}(\mathrm{z}=0)$ and the mean supernova neutrino energy for $5 \%$ background uncertainty. For $\left\langle\mathrm{E}_{\nu}\right\rangle \geq 14 \mathrm{MeV}$, a $3 \sigma$ detection is possible for the whole currently predicted range for the supernova rate. If the true supernova rate is in the upper part of the predicted range, even a $5 \sigma$ detection is possible for $\left\langle\mathrm{E}_{\nu}\right\rangle \geq 16 \mathrm{MeV}$.

In case that the number of detected events does not exceed the background rate, the current limit on the DSNB flux of the Super-Kamiokande experiment could be significantly improved. Assuming that 28 events are detected after $10 \mathrm{y}\left(\mathrm{N}_{\mathrm{det}}=\left\langle\mathrm{N}_{\mathrm{bg}}\right\rangle\right)$ and that the background is known with $5 \%$ uncertainty, the upper limit on the DSNB flux above $17.3 \mathrm{MeV}$ would be $0.4 \bar{\nu}_{\mathrm{e}} \mathrm{cm}^{-2} \mathrm{~s}^{-1}$ for $\left\langle\mathrm{E}_{\nu}\right\rangle=18 \mathrm{MeV}$, which is a factor of about 8 below the current limit [1].

Figure 9 shows the exclusion contours for the supernova rate $R_{\mathrm{SN}}(\mathrm{z}=0)$ and the mean supernova neutrino energy, assuming $5 \%$ background uncertainty and that no DSNB signal was detected. Independent of the mean supernova neutrino energy, the whole currently predicted range for the supernova rate would be covered by the $90 \%$ exclusion limit. The exclusion would be even at the $3 \sigma$ level if $\left\langle\mathrm{E}_{\nu}\right\rangle \geq 15 \mathrm{MeV}$. Hence, if no excess above the expected number of background events is found in LENA, all current standard DSNB models would be ruled out with more than $90 \%$ C.L.

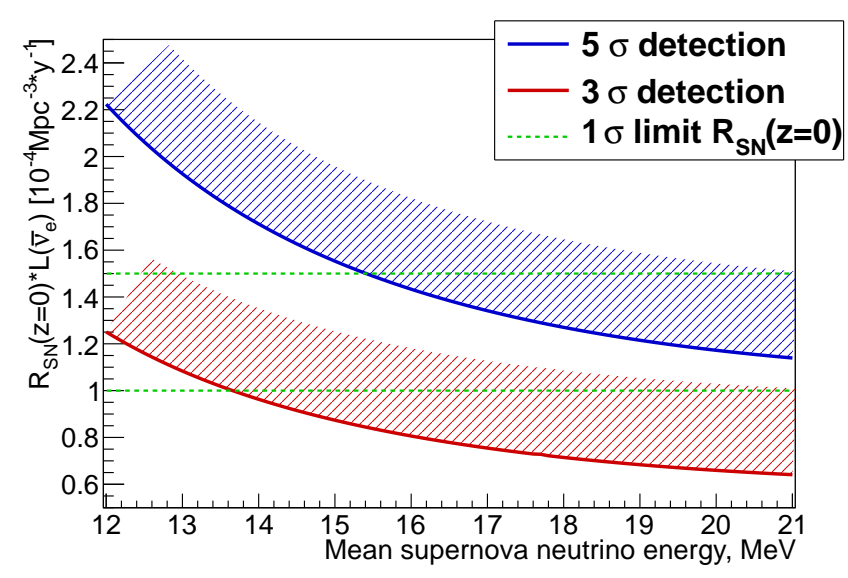

FIG. 8. The $3 \sigma$ and $5 \sigma$ detection potential for the DSNB, depending on the supernova rate $\mathrm{R}_{\mathrm{SN}}(\mathrm{z}=0)$ and the mean supernova neutrino energy for $5 \%$ background uncertainty and 10 years measuring time. For comparison, the current $1 \sigma$ confidence interval for $\mathrm{R}_{\mathrm{SN}}(\mathrm{z}=0)$ (green dashed lines) [12] is depicted.

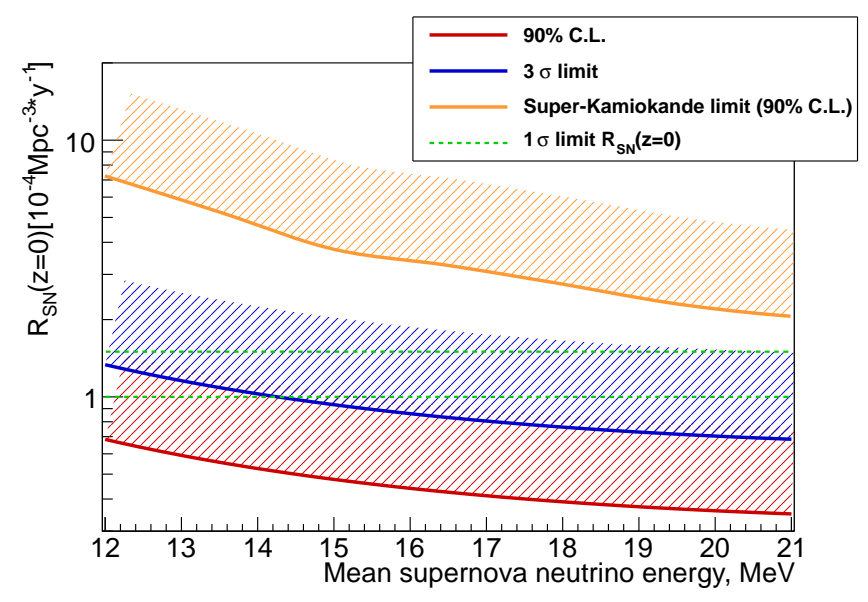

FIG. 9. The $3 \sigma$ (depicted in blue) and $90 \%$ C.L. (depicted in red) exclusion contours for the supernova rate $\mathrm{R}_{\mathrm{SN}}(\mathrm{z}=0)$ and the mean supernova neutrino energy, assuming $5 \%$ background uncertainty and no detected DSNB signal $\left(\mathrm{N}_{\mathrm{det}}=\left\langle\mathrm{N}_{\mathrm{bg}}\right\rangle\right)$. For comparison, the current $1 \sigma$ confidence interval for $\mathrm{R}_{\mathrm{SN}}(\mathrm{z}=0)$ (green dashed line) [12] and the $90 \%$ C.L. exclusion contours of the Super-Kamiokande experiment are depicted.

\section{CONCLUSIONS}

Due to its large target mass, the proposed LENA detector will be sensitive to the still undetected Diffuse Supernova Neutrino Background (DSNB). Indistinguishable background from reactor and atmospheric electron antineutrinos limit the detection window to neutrino energies from $9.5 \mathrm{MeV}$ to $25 \mathrm{MeV}$. Depending on the mean supernova neutrino energy, about 50 to 100 events per $10 \mathrm{y}$ are expected in this energy region. The most crucial 
background arises from neutral current reactions of atmospheric neutrinos, which can mimic the IBD event signature. It surpasses the DSNB signal by more than one order of magnitude. This background can be significantly reduced by a pulse shape analysis to $21.8 \pm 0.4$ (stat.) events per $10 \mathrm{y}$. Although this cut also reduces the DSNB event rate by $60 \%$, a signal to background ratio of around 1 is expected.

Assuming that the background rate is known at $5 \%$ uncertainty, LENA can detect the DSNB with $3 \sigma$ significance after $10 \mathrm{y}$. In case that no signal is found, the current upper limit on the DSNB flux can be improved by a factor of 8 . Furthermore, all current standard DSNB models could be ruled out with more than $90 \%$ C.L.

\section{ACKNOWLEGDEMENTS}

This research was supported by the DFG cluster of excellence 'Origin and Structure of the Universe' (Munich) and 'PRISMA' (Mainz).
[1] The Super-Kamiokande Collaboration, Phys. Rev. D85, 052007 (2012), arXiv:1111.5031.

[2] M. Wurm et al., Astroparticle Physics 35, 685 (2012), arXiv:1104.5620.

[3] M. Wurm et al., Phys. Rev. D75 023007 (2007), arXiv:astro-ph/0701305v1.

[4] The KamLAND Collaboration, Astrophys. J. 745, 193 (2012), arXiv:1105.3516.

[5] L. Oberauer, P. Pfahler, M. Wurm, "LAGUNA-LBNO Liquid Scintillator Specification Document," (2012), unpublished.

[6] V. A. Kudryavtsev, N. J. C. Spooner, J. E. McMillan, Nucl. Instrum. Meth. A505, 688 (2003), arXiv:hepex/0303007.

[7] A. Strumia, F. Vissani, Phys. Lett. B564, 42 (2003).

[8] R. Möllenberg, Monte Carlo Study of Solar ${ }^{8} B$ Neutrinos and the Diffuse Supernova Neutrino Background in LENA, Ph.D. thesis, Technische Universität München (2013).

[9] T. M. Undagoitia, Measurement of light emission in organic liquid scintillators and studies towards the search for proton decay in the future large-scale detector LENA, Ph.D. thesis, Technische Universität München (2008).

[10] S. Ando and K. Sato, New J. Phys. 6, 170 (2004), arXiv:astro-ph/0410061.

[11] E. E. Salpeter, Astrophys. J. 121, 161 (1955).

[12] S. Horiuchi, J.F. Beacom, E. Dwek, Phys. Rev. D79, 083013 (2009), arXiv:0812.3157.

[13] M. Wurm et al, Astroparticle Phys. 35, 685 (2012), arXiv: 1104.5620 .

[14] T. Yoshida et al., Astrophys. J. 686, 448 (2008), arXiv:0807.2723.

[15] M. T. Keil, G. G. Raffelt, H. T. Janka, Astrophys.J. 590, 971 (2003), arXiv:astro-ph/0208035v2.

[16] T. A. Thompson, A. Burrows, P. A. Pinto, Astrophys.J. 592, 434 (2003), arXiv:astro-ph/0211194.
[17] K. Sumiyoshi, S. Yamada, H. Suzuki, Astrophys. J. 667, 382 (2007), arXiv:astro-ph/0706.3762.

[18] S. Agostinelli et al., Nuclear Instruments and Methods 1A 506, 250 (2003).

[19] M. Wurm, Cosmic Background Discrimination for the Rare Neutrino Event Search in Borexino and LENA, Ph.D. thesis, Technische Universität München (2009).

[20] R. B. Firestone and V.S. Shirley (Ed.), Table of Isotopes, eight edition (John Wiley and Sons, 1996).

[21] C. Andreopoulos et al., Nucl. Instrum. Meth. A614, 87 (2010), arXiv:hep-ph/0905.2517.

[22] G.D. Barr, T.K. Gaisser, P. Lipari, S. Robbins, T. Stanev , Phys. Rev. D70, 023006 (2004), arXiv:astro$\mathrm{ph} / 0403630$.

[23] J. Winter, Neutron scattering in organic liquidscintillator and phenomenology of Supernova Neutrinos in LENA, Ph.D. thesis, Technische Universität München (2014).

[24] H.M. O'Keefe,E. O'Sullivan, M.C. Chen, Nucl. Instrum. Meth.A 460, 119 (2011), arXiv:1102.0797.

[25] S. Wagner, Ionization Quenching by Low Energy Electrons in the Double Chooz Scintillators, Diploma thesis, University of Heidelberg (2010).

[26] E. Litvinoch, I. Machulin, S. Silaeva, S. Sukhotin, Y. Suvorov, "Calibration of Borexino using the Am-Be source," (2009), internal Report.

[27] J. Meyer, Realization and Characterization of the Muon Veto Scintillator and the Buffer Liquid of the Double Chooz Experiment, Diploma thesis, Technische Universität München (2010).

[28] E. Gatti, F. De Martini, Nuclear Electronics vol. 2, 265 (1962).

[29] W. Rolke, A. Lopez, J. Conrad, F. James, Nucl. Instrum. Meth. A551, 493 (2005). 